\title{
Kiel 1969 als Erinnerungsort der deutschsprachigen Geographie - einige autoethnographische Anmerkungen
}

\author{
Peter Weichhart \\ Institut für Geographie und Regionalforschung, Universität Wien, Wien, Österreich \\ Correspondence: Peter Weichhart (peter.weichhart@univie.ac.at)
}

Published: 2 February 2021

„Wir erleben eine weltweite Konjunktur des Gedächtnisses. Seit zwanzig oder fünfundzwanzig Jahren macht sich allenthalben, in allen Ländern, allen gesellschaftlichen und ethnischen Gruppen eine tiefgreifende Veränderung der traditionellen Beziehung zur Vergangenheit bemerkbar.“ (Nora, 2002:18).

Mit dem Begriff des Erinnerungsortes wird die Vorstellung verknüpft, dass bestimmte Orte als historisch-kulturelle Bezugspunkte für bestimmte soziale Figurationen wirksam werden. Für Pierre Nora steht der Begriff des Erinnerungsortes stark mit der weltweiten „Konjunktur des Gedächtnisses“ in Verbindung. Dabei kann es sich um gesellschaftliche Megastrukturen (bei Nora geht es um die französische Nation), aber auch um Teilsegmente von Gesellschaften wie ethnische Gruppen oder kleinere Primärgruppen wie eine Familie handeln. Erinnerungsorte besitzen für die jeweilige Figuration eine hohe symbolische Bedeutung, markieren für sie historisch bedeutsame Sinnkontexte und besitzen damit eine identitätsverstärkende Funktion. Es wird angenommen, dass Erinnerungsorte zentrale Elemente des kollektiven Gedächtnisses (Halbwachs, 1967) der betreffenden Figuration darstellen. Das Erinnern an die gemeinsame Vergangenheit ist offensichtlich eines der Mittel, soziale Kohäsion auf verschiedenen Maßstabsebenen zu erzeugen. Erinnerungsorte verweisen dabei auf besonders dichte Konstellationen von Ereignissen, die aus der Perspektive der jeweiligen Gegenwart gleichsam als „Meilensteine“ der eigenen Entwicklung interpretiert werden. Sie werden damit als jene Elemente der Geschichte einer sozialen Figuration angesehen, derer man gedenkt und derer man gedenken sollte.

Ist „Kiel 1969“ ein Erinnerungsort für die soziale Figuration der deutschsprachigen Geographinnen und Geographen? Zunächst ist anzumerken, dass Erinnerungsorte nicht aus sich selbst heraus existieren. Sie wachsen nicht wie Bäume oder Pilze, sondern sie werden gemacht. Wenn wir etwas „ins Gedächtnis rufen“, dann ist das eine bewusste Anstrengung, ein aktives Handeln. Erinnerung ist nicht einfach da, sondern muss geweckt oder aktualisiert werden. Vor allem die Bedeutungszuschreibung, die Aufladung eines vergangenen Ereignisses mit bestimmten Sinnkontexten und Konstellationen des Geschehens ist als aktive und bewusste Interpretationsleistung zu verstehen. Dahinter stehen spezifische Akteur*innen, die damit für sich auch gewisse Interpretationsprivilegien beanspruchen oder behaupten.

Aus den vorliegenden Texten und Kommentaren kann abgeleitet werden, dass bereits die Zeitgenoss*innen des Geschehens und die Teilnehmer*innen des Kieler Geographentages dieses Ereignis als etwas Besonderes und Bedeutsames wahrgenommen und bewertet haben. Zwar wurde die Veranstaltung von manchen Fachvertreter*innen und Teilnehmer*innen sehr zurückhaltend und mit deutlichem Understatement beschrieben und bewertet, dennoch machen viele Wortmeldungen auch im Umfeld der Veranstaltung deutlich, dass Kiel 1969 schon unmittelbar danach als besonderes Ereignis angesehen wurde (vergl. Weichhart, 2016:9).

In den Folgejahren wurden von theoretisch interessierten Geograph*innen immer wieder Verweise auf diesen Geographentag getätigt, in denen seine Bedeutung für das Fach ausdrücklich betont wurde. Die Autor*innen solcher Beiträge wurden damit zu Begründer*innen und Träger*innen spezifischer Interpretationen der Fachgeschichte, welche die Grundlagen dafür darstellen, dass Kiel in der Folge zu einem Erinnerungsort werden konnte. „Kiel wurde zu ,Kiel ${ }^{\text {‘ }}$ gemacht, die Bedeutung, die ,Kiel` für die deutschsprachige Geographie hat, ist Folge einer aktiven Arbeit am Mythos (Michel, 2014: 301, vergl. dazu auch Strohmayer, 2020).

Die dabei entstandenen Narrative unterscheiden sich allerdings durchaus in der Gewichtung einzelner Konsequenzen des Ereignisses, aber auch in den Schwerpunkten der (Über-)Generalisierungen, die bei den fachhistorischen Inter- 
pretationen vorgenommen wurden. Mit anderen Worten: Was inhaltlich von den verschiedenen Kommentator*innen dieses Ereignisses der Fachgeschichte als bedeutsam herausgestellt wurde (und wird), weist ein sehr breites Spektrum von zum Teil widersprüchlichen Deutungsmustern und Behauptungen auf. Der Erinnerungsort „Kiel““ ist also mit sehr unterschiedlichen Konnotationen und Assoziationen verknüpfbar.

Das macht es für die für die jüngeren Kohorten der Geograph*innen, die schon aus Altersgründen nur auf dem Weg der fachlichen Sozialisation mit den Ereignissen und Konsequenzen von Kiel 1969 in Kontakt kommen, sehr schwierig, subjektiv ein klares Bild dieses „Meilensteins“ der Fachgeschichte entwerfen zu können. Das Einzige, worüber bei den „Hüter*innen“ und Erzeuger*innen des Erinnerungsortes offensichtlich Einigkeit besteht, ist die Auffassung, dass Kiel 1969 als signifikantes Ereignis, also eben als Meilenstein, aufgefasst werden müsse. $\mathrm{Ob}$ dieser Meilenstein nun als Leuchtturm oder Irrlicht (Werlen, 2014), als Ermöglichung für Praxisrelevanz und für Berufsoptionen außerhalb der Schule (Monheim et al., 1999), als Signal für den Umbau des Faches in Richtung auf Quantifizierung und Modellbildung (Arnreiter und Weichhart, 1998), als Öffnung der Geographie für kritische gesellschaftstheoretische Ansätze (Schurr und Weichhart, 2020:56) oder als vergebliches Bemühen anzusehen ist, das Fach aus dem minderen Status der ,folk science“ herauszuheben und weiterzuentwickeln (Hard, 1979), hängt also von den Interpretationen der jeweiligen „Hüter*innen“ und Produzent*innen des Erinnerungsortes ab.

Die Narrative zu Erinnerungsorten gründen auf der Bedeutung und Relevanz, die von den Hütern und Hüterinnen behauptet werden. Den Adressaten und Adressatinnen wird dies auf dem Wege über Sozialisationsprozesse vermittelt. Im Falle von Kiel sind die Adressat*innen natürlich die Vertreter*innen der Figuration der Geographinnen und Geographen. Aber handelt es sich hierbei tatsächlich um eine kompakte und abgrenzbare Figuration? Diese Frage stellt sich allerdings für jeden Erinnerungsort. Auch die von Nora angesprochene ,französische Nation“ lässt sich wohl kaum auch nur einigermaßen abgrenzen und inhaltlich bestimmen. Es scheint vielmehr umgekehrt zu sein: Erst durch die Konstruktion von Erinnerungsorten und die Verweise auf eine gemeinsame Geschichte und gemeinsame Traditionen entsteht so etwas wie ein gesellschaftliches System oder Subsystem, für das kollektive Identität konstruierbar wird. Für die Geographie bedeutet das, dass bei den einzelnen Fachvertreter*innen ganz unterschiedliche inhaltliche Attributierungen mit dem Erinnerungsort „Kiel“ verknüpft werden, abhängig von Alterskohorten, Studienort, fachlicher Spezialisierung, fachhistorischen Interessenlagen und Neigung zur Reflexion geistesgeschichtlicher Zusammenhänge. Der hohen Inhomogenität bei der Produktion der Narrative steht also eine zumindest gleich große Inhomogenität ihrer individuellen Rezeption gegenüber.
„Fest steht: Geschichte passiert nicht an einem Tag““ (Schlottmann und Wintzer, 2019:178). Mit dieser Aussage kommentieren die Autorinnen das singuläre Ereignis des Kieler Geographentages 1969. Eine gängige Attribuierung für den „Mythos Kiel“", die in vielen Überlegungen zur Fachgeschichte zumindest implizit zum Ausdruck kommt, ist ja die Vorstellung, dass mit dem Geographentag eine schlagartige Wende für die Geographie eingesetzt hätte. Vor Kiel habe es die ,klassische Geographie“ gegeben, nach Kiel hätte eine völlige Neuorientierung begonnen. Von verschiedenen Autor*innen wurde zurecht darauf hingewiesen, dass es sich bei dieser Behauptung um eine wohl auch didaktisch motivierte Übergeneralisierung handelt (vgl. z. B. auch Michel, 2014). Kiel hatte ein sehr langes ,Vorspiel“", mit dem die Bühne für die „Revolution“ ausführlich vorbereitet wurde (vgl. Wardenga, 2020). Und nach Kiel änderte sich für das Fach längere Zeit gar nicht viel.

Am Deutschen Kongress für Geographie 2019 (ich tendiere immer noch dazu, einfach vom „Geographentag“ zu sprechen) wurde unter dem Titel „Kiel 1969: Ein Erinnerungsort der Geographie“ eine von Benedikt Korf und Ute Wardenga geleitete Podiumsdiskussion veranstaltet, zu der als Panellist*innen Julia Verne, Ulf Strohmayer und der Autor geladen waren. Uns wurden von der Sitzungsleitung im Vorfeld einige Fragen vorgelegt, auf die wir im Podiumsgespräch eingehen sollten. Die erste Frage bezog sich darauf, in welchem Zusammenhang wir in unserer fachlichen Sozialisation das erste Mal über „Kiel 1969“ „gestolpert“ seien.

Ich war der einzige in dieser Runde, der - vom Lebensalter her gesehen - beim Geographentag 1969 in Kiel hätte dabei sein können. Nein, ich war aber auch nicht dort. Ich war damals im fünften Semester meines Geographiestudiums an der Universität Salzburg. Ich bin aber dennoch sehr zeitnah über die Ereignisse dieses Events ,gestolpert“, denn mein späterer Doktorvater, der damals gerade frisch berufene Physiogeograph Helmut Riedl, hatte in seiner Vorlesung den Studierenden einen sehr ausführlichen Bericht über den Kieler Geographentag vorgetragen. Ich habe diese Geschichte schon mehrfach erzählt (Weichhart, 2016:7), will sie aber doch noch einmal zusammenfassen, weil sie für mich ausgesprochen bedeutsam war und die damalige Situation im Fach recht gut charakterisiert.

Riedls Bericht war überaus dramatisch formuliert. In Kiel hätten rebellierende Studierende und einige jüngere Dozenten einen Putschversuch unternommen, bei dem die Grundlagen der Geographie erschüttert werden sollten. Die für das Fach konstitutive Einheit, also die untrennbare Verknüpfung von Physio- und Humangeographie, solle aufgelöst werden, die Länderkunde sei unwissenschaftlich, und das Landschaftskonzept, das methodologische Zentrum und der zentrale Forschungsgegenstand der Geographie, sei zu verwerfen. Eine Umsetzung dieser Forderungen würde die Geographie als Wissenschaft nicht nur in ihren Grundfesten erschüttern, sondern dieses Fach einfach zerstören. Selbstverständlich hätte aber der weitaus überwiegende Teil der Fachvertre- 
ter*innen diese unsinnigen Forderungen dezidiert abgelehnt und als Unfug entlarvt.

Riedls Bericht hatte mich neugierig gemacht. Denn auch in meinem zweiten Fach, der Germanistik, gab es damals Grundsatzdiskussionen über den Zustand und die weitere Entwicklung der Disziplin. Da ich seit Beginn meines Studiums auch Lehrveranstaltungen in der Philosophie belegt hatte - zum Beispiel Wissenschaftstheorie bei Paul Weingartner -, hatte ich damals bereits realisiert, dass es in den Wissenschaften immer wieder Grundlagenkrisen und revolutionäre Umbrüche gibt. Und weil ich schon in den ersten Semestern Schwierigkeiten mit den Grundkonzepten der klassischen Geographie hatte - die Länderkunde fand ich ziemlich öde und wenig erhellend, die Logik des Landschaftskonzepts leuchtete mir gar nicht ein -, wollte ich der Sache näher auf den Grund gehen. Ich begann also, mich ernsthaft mit den Schriften der „Revolutionäre“ zu befassen. Ich vertiefte mich in die Habilitationsschriften von Bartels und Hard, die mich sehr beeindruckten, und beschäftigte mich mit der Paradigmenforschung der Philosophie. Dabei war es mir ein Anliegen, deren Erkenntnisse auf das Fach Geographie anzuwenden.

Vor allem aber war dies ein Anstoß, mich mit den theoretischen und methodologischen Grundlagen der klassischen Geographie auseinanderzusetzen. Ich befasste mich also sehr intensiv mit den Schriften der wichtigsten Vertreter der klassischen Geographie und versuchte, die von ihnen entwickelten theoretischen Grundlagen zu rekonstruieren. Schon in den Lehrveranstaltungen des ersten Studienabschnitts war mir aufgefallen, dass von den Vortragenden sehr dezidierte dogmatische Aussagen zu den Themen „Länderkunde“, „Landschaft“ und „Einheit der Geographie“ gemacht wurden, mir auf konkrete Nachfragen und Begründungswünsche aber nur sehr vage und unklar geantwortet wurde. Jetzt wollte ich es also genauer wissen und bemühte mich, Antworten auf meine Fragen bei den großen Klassikern selbst zu finden: „Was genau bitte ist eine Landschaft?“ „Wie funktioniert die Integration der Geofaktoren?“" „Was sind eigentlich Geofaktoren?“" „Warum ist die Einheit des Faches und die unaufhebbare Verbindung von Humangeographie und Physiogeographie so wichtig?“ „Warum ist die Länderkunde die Königsdisziplin des Faches?"

Mich selbst und meine eigene Arbeit hat Kiel also sehr entscheidend beeinflusst. Einerseits war ich vom Streben der „Revolutionäre“ nach einer wissenschaftstheoretischen Grundlegung des Faches und der Betonung methodologischer Reflexionen sehr angetan, andererseits fand ich die Suche nach nomologischen Theorien höchst anregend. In den Schriften von Bartels und Hard fand ich erstmals in der deutschsprachigen Geographie die Auffassung, dass Wissenschaft nur mit Hilfe und auf der Grundlage von Theorien betrieben werden könne. Vor dem Hintergrund der Wissenschafts- und Erkenntnistheorie war mir das bereits klar. Ich war auch beeindruckt von der Präzision der Begriffsbestimmungen und der analytischen Schärfe bei der Dekon- struktion geographischer Grundkonzepte in den Arbeiten von Bartels und Hard.

Sehr bald regte sich bei mir aber auch Widerspruch zu einigen Grundthesen der Kieler Revolution. So konnte ich schon damals nicht nachvollziehen, dass mit der Hinwendung zum Neopositivismus bzw. logischen Empirismus die wissenschaftstheoretische Grundlegung einer Geographie des Menschen gefunden worden sei. Auch die Fokussierung auf chorologische Theorieansätze erschien mir zu einseitig. Ich hätte mir nicht nur mehr Gesellschaftstheorie, sondern auch eine ausdrückliche Einbindung von Theorien mittlerer Reichweite aus Nachbardisziplinen wie Soziologie und Psychologie gewünscht. Vor allem aber vermisste ich Hinweise auf Möglichkeiten einer Theorie der Mensch/Gesellschaft-UmweltBeziehungen. Ich begann also, mich intensiv mit Ökologie und vor allem mit Human- und Sozialökologie zu beschäftigen.

Besonders hilfreich für mich war aber auch die intensive Auseinandersetzung mit den Schriften der „Klassiker“ (vgl. Weichhart, 1975). Aus der Lektüre zahlreicher Texte von Autoren wie Hans Bobek, Harald Uhlig, Josef Schmithüsen, Hermann Lautensach, Ernst Winkler, Ernst Neef und vieler anderer bot sich mir ein völlig anderes und wesentlich differenzierteres Bild der klassischen Geographie, als ich es in den von mir besuchten Lehrveranstaltungen vermittelt bekommen hatte. Dabei musste ich erstens erkennen, dass die klassische Geographie keineswegs ,theorielos“ war, sondern sich in den einschlägigen methodologischen Arbeiten sehr ernsthafte und komplexe Ansätze einer theoretischen Reflexion finden. Zweitens wurde mir im Zeitverlauf immer deutlicher klar, dass viele Autor*innen, die später und bis heute ablehnend und abwertend über diese Phase der Geographie geschrieben haben, die Klassiker entweder nicht allzu häufig im Original gelesen haben können oder ihre Informationen nur aus zweiter Hand bezogen haben. Drittens zeigte sich, dass die klassische Phase keineswegs so homogen und gleich ,getaktet“ war, wie dies häufig behauptet wird. Viele der Kritikpunkte, die in der Diskussion um Kiel öffentlich und an prominenter Stelle artikuliert wurden, hatten bereits die Klassiker reflektiert, prominent publiziert und durchaus als offene Fragen oder ungelöste Probleme dargestellt. Das Krisenbewusstsein wurde bereits lange vor Kiel sehr deutlich angesprochen (vergl. Schultz, 1980:31-39, Arnreiter und Weichhart, 1998:54-56; Weichhart, 1975).

Mit all diesen Überlegungen hatte ich - gleichsam zwanglos - mein Dissertationsthema gefunden. Diese Arbeit wurde 1973 unter dem Titel „Methodologische Probleme der Geographie“ approbiert. Der Titel der 1975 erschienenen Druckfassung „Geographie im Umbruch“ stellte dann aber deutlicher heraus, dass es mir um eine inhaltliche Aufarbeitung von wesentlichen Aspekten der „Kieler Wende“ ging. Ganz besonders wollte ich dabei auch Überlegungen anstellen, wie man in der Geographie nach Kiel das Gesellschaft-UmweltProblem angemessen thematisieren könnte. 
Auch viele meiner späteren Arbeiten und Projekte - etwa im Rahmen der Paradigmenforschung - bezogen sich direkt oder indirekt auf die Ereignisse von Kiel 1969. Ich kann also sagen, dass dieser Geographentag vor mehr als 50 Jahren und sein Umfeld meine eigene wissenschaftliche Arbeit für viele Jahre grundlegend beeinflusst hat - womit aus meiner Sicht auch die zweite Frage beantwortet ist, die uns Panellist*innen von der Sitzungsleitung gestellt worden war.

Weiters sollten wir in unseren Ausführungen auf dem Podium darlegen, welche Erzählungen von Kiel 1969 wir in welchen Diskurskontexten wahrgenommen hätten. In meinem damaligen fachlichen Umfeld war es eigentlich nur die oben bereits angesprochene „Bedrohungs- und Untergangserzählung“, die aber lediglich für relativ kurze Zeit aktuell war. Schließlich hätten sich die Vernünftigen im Fach ja durchgesetzt und die Bedrohung,,abgewehrt“. Auch von studentischer Seite wurde das Thema nicht vertieft. Sozialtheorie war damals im Kontext meiner persönlichen fachlichen Sozialisation einfach kein Thema. (Die 68er-Bewegung hatte in der Provinzstadt Salzburg keine besonders auffällige Bedeutung - jedenfalls nicht an der naturwissenschaftlichen Fakultät, zu der die Geographie gehörte.)

Bei meinen Lehrer*innen und Kommiliton*innen konnte ich also auch in den folgenden Jahren kein wirkliches Interesse an den Ereignissen von Kiel feststellen. Es war weiterhin „,business as usual“ angesagt, es gab keine Änderungen der Lehrinhalte oder Curricula. Kiel ist an der damaligen Salzburger Geographie eigentlich spurlos vorübergegangen. Im Verlaufe meines Geographiestudiums hatte ich mich bemüht, länderkundliche Lehrveranstaltungen möglichst zu vermeiden. Dennoch musste ich insgesamt 32 Wochenstunden zum Thema „Länderkunde von ... “ absolvieren. An eine der Prüfungsfragen beim Kolloquium zur „Länderkunde von Österreich“ erinnere ich mich noch genau: „Sie fahren auf der Autobahn von Salzburg nach Wien. Nennen Sie alle Abfahrten auf dieser Strecke.“

Ähnliches musste ich später auch für das Geographieinstitut der Münchener LMU feststellen. Ganz anders war die Situation an der TU München (vergl. Strohmayer, 2020), das räumlich nur wenige Schritte entfernt lag. Die soziale und fachliche Distanz zwischen beiden Instituten war demgegenüber nur in Lichtjahren zu messen - jedenfalls zu meiner Zeit in München (1975 bis Anfang 1980). Obwohl ich von einem Kollegen am LMU-Institut ausdrücklich gewarnt wurde, dass Kontakte mit dem anderen Institut gar nicht opportun seien, besuchte ich regelmäßig Kolloquiumsvorträge an der TU und kam natürlich mit den dortigen Kolleg*innen ins Gespräch. Ich habe diese Interaktionen auch vertieft und zahlreiche Diskussionen geführt, besonders mit Heiner Dürr, Robert Geipel, Sabine Tzschaschel und Jürgen Pohl. Ich durfte auch als Gast an Seminaren von Robert Geipel teilnehmen. An diesem Institut war Kiel durchaus ein wichtiges Thema, und hier stand die „Fortschritts- und Erneuerungsnarration“ im Zentrum. Negative Konsequenzen oder Sanktionen am LMU-Institut hatte ich wegen dieser Kontakte nicht zu tra- gen, dafür war ich in der Hackordnung des Hauses einfach zu unwichtig. Herr Gierloff-Emden, damals geschäftsführender Institutsvorstand, hat mich allerdings einmal am Gang angesprochen: „Wie ich höre, kooperieren Sie jetzt mit dem Feind."

Für Herrn Gierloff-Emden waren besonders auch die „,Revolutionäre“ von Kiel „Feinde“ (Weichhart, 2016:8). Mit dem Physiogeographen Friedrich Wilhelm, dem zweiten Ordinarius am LMU-Institut, habe ich mehrfach versucht, Gespräche über Kiel zu führen. Er war an diesem Thema aber einfach nicht interessiert. Der einzige Lichtblick an diesem Institut war diesbezüglich mein direkter Vorgesetzter, Helmut Heuberger. Er war international vor allem durch seine Forschungen zum Spätglazial bekannt, war aber auch im Bereich der ,alten Kulturgeographie“ aktiv. Er hat mich bei meinen methodologischen Bemühungen ausdrücklich unterstuitzt und dazu ermuntert. Seine Position zu Kiel war die eines ,Erkenntnisdarwinisten“ (Arnreiter und Weichhart, 1998:76-78): „Lasst die Leute nur machen. Es wird sich schon zeigen, was davon brauchbar ist."

Im Februar 1980 beendete ich meine Tätigkeit in München und trat eine Stelle als Assistent an der Geographie in Salzburg an. Meine Frau und ich waren über diesen Wechsel sehr glücklich, denn insgesamt waren die fünf Jahre in München für uns ein beinahe traumatisches Erlebnis. Im März 1980 wir hatten gerade unsere neue Wohnung in Salzburg bezogen - erhielt ich einen Anruf aus Kiel. Es war Dietrich Bartels, der mir nach kurzem Smalltalk eine Assistentenstelle bei ihm anbot. Meine Publikationen hätten ihn beeindruckt, und er würde mich gerne als Mitarbeiter begrüßen. Ich fand dieses Angebot natürlich sehr aufregend und ehrenvoll, lehnte es aber mit dem Hinweis auf unsere eben erfolgte Übersiedlung und die neue Stelle spontan ab. So hätte meine inhaltliche Beschäftigung mit Kiel 1969 letztlich beinahe dazu geführt, dass ich als Assistent eines der führenden „Revolutionäre“" an das Geographieinstitut in Kiel gekommen wäre.

Meine Versuche, die Kolleg*innen am neuen Arbeitsplatz in Salzburg von der Notwendigkeit der Theoriearbeit zu überzeugen, scheiterten kläglich. ,Theoretisch“ bedeutete für sie einfach nur ,nicht wirklich“. Helmut Riedl sagte einmal zu mir: „Hören Sie doch auf, diese vielen Bücher zu lesen. Gehen Sie hinaus und lassen Sie sich den Wind des Geländes um Ihre grünen Ohren blasen!“ Kiel war auch 1980 und in den Folgejahren in der Salzburger Geographie kein Thema.

Ich habe mich sehr oft und intensiv mit Kolleg*innen aus der Physischen Geographie über Kiel unterhalten. Für viele von ihnen ist Kiel 1969 eher bedeutungslos und ohne Relevanz für das eigene Selbstverständnis. Manche von ihnen sehen Kiel jedoch eher positiv. Wenn ich deren Meinung - etwas überpointiert - zusammenfasse, dann könnte man das so formulieren: „Gut, dass nach Kiel diese enge Bindung zur Humangeographie aufgegeben wurde. Damit konnte sich die Physiogeographie endlich zu einer richtigen (Natur-)Wissenschaft weiterentwickeln. Wir sind diese 
lästige Nabelschau und kritische Selbstreflexion endlich los und können uns auf die wirkliche und eigentliche wissenschaftliche Arbeit konzentrieren. Und wir können über die Natur forschen, ohne ständig darüber nachdenken zu müssen, was das eigentlich ist, und ob es sie eigentlich wirklich gibt.“ Das war natürlich kein wörtliches Zitat, sondern eine überspitzte Zusammenfassung meiner Wahrnehmung dieser Gespräche. Eine aus meiner Sicht etwa gleich große Gruppe (vorwiegend älterer) Physiogeograph*innen bedauerte allerdings den Bruch und sahen ihn als Traditionsverlust. Wirklich überzeugende Begründungen für ihre Auffassung habe ich allerdings nie gehört.

Auch mit Vertreter*innen der Humangeographie habe ich in den Jahren nach Kiel zahlreiche Gespräche über den Status des Faches geführt. Dabei hatte ich überwiegend den Eindruck, dass die Auflösung der Einheitsgeographie durchaus positiv gesehen wurde. Man könne sich nun endlich und ohne Rechtfertigungsdruck mit den jeweiligen Spezialthemen (wie Einzelhandelsforschung, Verkehrssystemen oder Fremdenverkehr) befassen, ohne nach Metabegründungen zur Einbindung in das „Gesamtfach“ suchen zu müssen.

Eine weitere Frage der Sitzungsleitung an die Panellist*innen lautete: „Wofür steht Kiel 69 aus meiner Sicht in der disziplin-politischen Auseinandersetzung? Wofür oder wogegen wird ein spezifischer Diskurs zu Kiel 69 mobilisiert?“

Meiner Wahrnehmung nach stand Kiel während der letzten zwei bis drei Jahrzehnte in der disziplinpolitischen Auseinandersetzung nicht besonders auffällig im Vordergrund. Wesentlich präsenter war im deutschen Sprachraum etwa die Frage nach der Positionierung der Neuen Kulturgeographie und davor die Auseinandersetzung mit der handlungszentrierten Sozialgeographie. Mobilisiert - und zwar in negativem Sinne - wurde Kiel 69 bei den verschiedenen Versuchen, eine Reintegration der beiden Geographien zu beschwören. Ich vermute, dass nur das Faktum des fünfzigjährigen Jubiläums dazu geführt hat, dass man kurz davor begonnen hatte, wieder an Kiel 1969 zu denken. Nach zahlreichen Gesprächen mit jüngeren und älteren Geograph*innen habe ich den Verdacht, dass Kiel 69 für viele als weitgehend „vergessene“ Geschichte anzusehen ist.

Und gerade deshalb denke ich - und das ist meine Antwort auf die letzte Frage an uns -, dass die Erinnerung an Kiel auch heute noch sinnvoll ist. Carolin Schurr und ich haben bei den Recherchen zu unserer Keynote-Lecture (Schurr und Weichhart, 2020) mit Erstaunen festgestellt, dass es so gut wie keine Dokumente oder Archivalien zu Kiel 69 gibt. Was fehlt, ist eine solide und professionelle Aufarbeitung der Ereignisse von Kiel und des gesamten geistesgeschichtlichen und fachpolitischen Kontextes durch Fachhistoriker.

So geht Wardenga (2020:5) davon aus, dass sich das Geschehen weitaus besser verstehen lässt, ,... wenn man die damals handelnden Akteure und ihre jeweiligen Ziele auch vor einem weiteren zeithistorischen Hintergrund betrachtet". Sie verweist darauf, dass in der frühen Nachkriegszeit auch im Fach Geographie eine langwierige Aufbauleistung für die Schaffung der notwendigsten Infrastruktur erforderlich war, was auch dazu führte, dass man in Lehre und Forschung jahrelang ,... auf das vorhandene Material und damit auf die vertrauten Konzepte von Länder- und Landschaftskunde aus der Vorkriegszeit zurückgriff“" (Wardenga, 2020:8). Zukunft war für die damals aktiven Hochschulgeographen, die wegen der gemeinsamen Not der Unterversorgung als homogene soziale Gruppierung agierten, ,,vorwiegend traditional, mithin als Erwartungshorizont einer bruchlosen Kontinuierung von Herkunft vorstellbar" (Wardenga, 2020:9).

Wardenga merkt ferner an, dass Anfang der 60er-Jahre das Thema „Entwicklungspolitik“ hochaktuell war. Die Hochschulgeographie sah darin eine hervorragende Möglichkeit, sich unter Berufung auf ihre Kompetenz in der Länderkunde öffentlichkeitswirksam zu profilieren und deshalb den Ausbau der institutionell und personell unterentwickelten Geographie zu fordern. Denn die Geographie könne als ,... länderkundlich ausgerichtete Basisdisziplin für die politisch immer wichtiger werdende Entwicklungsländerforschung“" (Wardenga, 2020:10) eine bedeutsame Rolle spielen. Derartige Überlegungen können erklären, warum auch viele der als „fortschrittlich“ eingeschätzten Hochschullehrer die Länderkunde - aller methodologischen Probleme zum Trotz - als Zukunftsperspektive des Faches eingeschätzt haben.

Auch Wardenga stellt fest, dass sich die inhaltliche und organisatorische Vorbereitung des Kieler Geographentages nicht mehr exakt nachverfolgen lässt, denn entsprechende Unterlagen fehlen in den Archiven. Dennoch kann sie im letzten Abschnitt ihres Artikels einige Aspekte dieses bedeutsamen Ereignisses rekonstruieren (Wardenga, 2020:1620) und dabei deutlich machen, dass der Geographentag in Kiel 1969 als Ergebnis einer sehr komplexen und von vielen Akteur*innen gesteuerten Inszenierung anzusehen ist.

Ja, die Erinnerung an Kiel 1969 erscheint mir auch heute noch sinnvoll. Gerade eine solide fachhistorische Aufarbeitung des Geschehens und seines Kontextes vermag uns daran erinnern, dass Wissenschaft nicht nur ein als kognitive Struktur beschreibbares Denkgebäude darstellt, sondern immer auch als spezifische soziale Praxis und als komplexes soziales System verstanden werden muss (Wardenga und Weichhart, 2012:299).

Die Erinnerung an Kiel sollte auch ein Fixpunkt in der Grundausbildung des Geographiestudiums sein. Ich halte es für ,nahrhaft" und wichtig, dass Studierende wenigstens in groben Zügen die geistesgeschichtliche Entwicklung des eigenen Faches präsent haben. Die Erinnerung an Kiel 69 kann in der Lehre auch als Musterbeispiel für einen Paradigmenwandel herangezogen werden. Und von diesem Modell aus ließe sich auch die aktuelle multiparadigmatische Struktur unserer Wissenschaft anschaulich aufzeigen.

Datenverfüg barkeit. Für diesen Artikel wurden keine Datensätze genutzt. 
Interessenkonflikt. Der Autor erklärt, dass kein Interessenkonflikt besteht.

\section{Literatur}

Arnreiter, G. und Weichhart, P.: Rivalisierende Paradigmen im Fach Geographie, in: Koexistenz rivalisierender Paradigmen. Eine post-kuhnsche Bestandsaufnahme zur Struktur gegenwärtiger Wissenschaft, Herausgeber: Schurz, G. und Weingartner, P., Westdeutscher Verlag, Opladen und Wiesbaden, 53-85, 1998.

Halbwachs, M.: Das kollektive Gedächtnis, Enke, Stuttgart, 1967.

Hard, G.: Die Disziplin der Weißwäscher, in: Zur Situation der deutschen Geographie zehn Jahre nach Kiel, Herausgeber: Sedlacek, P., Selbstverlag des Fachbereichs 2 der Universität, Osnabrück, 11-44, 1979.

Michel, B.: Wir sind nie revolutionär gewesen - Zum Mythos des Kieler Geographentags als der Geburtsstunde einer neuen Geographie, Geogr. Helv., 69, 301-303, https://doi.org/10.5194/gh69-301-2014, 2014.

Monheim, H., Schwarte, M. und Winkelkötter, C.: Die deutsche Geographie dreißig Jahre nach Kiel. Stadt-Land-Fluß oder ernstzunehmende Raumwissenschaft? Standort, Zeitschrift für Angewandte Geographie, 3, 46-49, 1999.

Nora, P.: Gedächtniskonjunktur, Transit, 22, 18-32, 2002.

Schlottmann, A. und Wintzer, J.: Weltbildwechsel. Ideengeschichten geographischen Denkens und Handelns, Haupt Verlag, Bern, (= utb 5218), 2019.
Schultz, H.-D.: Die deutschsprachige Geographie von 1800 bis 1970. Ein Beitrag zur Geschichte ihrer Methodologie, Berlin (= Abhandlungen des Geographischen Instituts, Anthropogeographie 29), 1980.

Schurr, C. and Weichhart, P.: From Margin to Center? Theoretische Aufbrüche in der Geographie seit Kiel 1969, Geogr. Helv., 75, 53-67, https://doi.org/10.5194/gh-75-53-2020, 2020.

Strohmayer, U.: An was genau erinnert „Kiel 1969“? , Geogr. Helv., 75, 215-219, https://doi.org/10.5194/gh-75-215-2020, 2020.

Wardenga, U.: Vergangene Zukünfte - oder: Die Verhandlung neuer Möglichkeitsräume in der Geographie, Geogr. Z., 108, 4-22, 2020.

Wardenga U. und Weichhart, P.: Entzauberung und Skandalisierung von Wissenschaft oder Reflexion des Normalzustandes einer sozialen Praxis?, Berichte zur deutschen Landeskunde, 86, 297308, 2012.

Weichhart, P.: Geographie im Umbruch. Ein methodologischer Beitrag zur Neukonzeption der komplexen Geographie, Franz Deuticke, Wien, 1975.

Weichhart, P.: Der Kieler Geographentag 1969 - Modernisierungsschub, Mythos, Paradigmenwandel oder vergessene Geschichte?, Geogr. Helv., 71, 7-13, https://doi.org/10.5194/gh-71-7-2016, 2016.

Werlen, B.: Kiel 1969 - Leuchtturm oder Irrlicht?, Geogr. Helv., 69, 293-299, https://doi.org/10.5194/gh-69-293-2014, 2014. 